AperTO - Archivio Istituzionale Open Access dell'Università di Torino

Evolutionary mechanisms and neural adaptation: Selective versus constructive strategies in the development and plasticity of the nervous system

This is a pre print version of the following article:

Original Citation:

Availability:

This version is available http://hdl.handle.net/2318/89330

since

Publisher:

Springer

Terms of use:

Open Access

Anyone can freely access the full text of works made available as "Open Access". Works made available under a Creative Commons license can be used according to the terms and conditions of said license. Use of all other works requires consent of the right holder (author or publisher) if not exempted from copyright protection by the applicable law. 


\section{Evolutionary Mechanisms and Neural Adaptation: Selective Versus Constructive Strategies in the Development and Plasticity of the Nervous System}

Ferdinando Rossi

Abstract The correct function of the nervous system requires complex neural 6 networks bearing precise connections. In principle, the high structural specificity 7 of neural circuits could be achieved by genetically-determined processes, selected 8 and refined during evolution. Highly conserved gene networks regulate some 9 crucial steps of neural development, such as the regionalization of the neural tube 10 and the initial phases of neurogenesis and synaptogenesis. A totally hardwired 11 nervous system may meet the requirements of adaptation and natural selection at 12 the population level. Nevertheless, it would be inadequate to allow individual 13 organisms to cope with rapid changes of environmental conditions. Neural adapta- 14 tion to external constraints can be partly achieved by introducing selective 15 mechanisms in neural development. Accordingly, neurons are generated in excess 16 and then partially eliminated to match the actual extension of innervation 17 territories. Such mechanisms, however, are restricted to a set of potentialities, 18 which must be predetermined in the ontogenetic program. On the other hand, 19 constructive mechanisms, in which external stimuli directly influence structural 20 modifications of neural circuits to produce adaptive responses, may allow individ- 21 ual organisms to cope with a wide variety of unprecedented situations. Thus, in the 22 last ontogenetic period as well as in the adult, when the organism actively interacts 23 with the external milieu, experience exerts a strong growth-promoting effect on 24 neural circuits and connections inducing the emergence of specific functional 25 properties. By this mechanism, which requires strict inhibitory control to prevent 26 aberrant growth and dysfunction, the nervous system exploits external stimuli to 27 create adaptive responses to unexpected situations.

F. Rossi ( $\square)$

Department of Neuroscience, Section of Physiology, University of Turin, Orbassano, Torino, Italy e-mail: ferdinando.rossi@unito.it 
Over the last decades, substantial advancements have been obtained in the elucidation of the cellular and molecular interactions that regulate the development of the nervous system, govern its function and determine its plastic capabilities in adulthood. These discoveries have led to the proposition of concepts and principles that relate, in a very peculiar manner, developmental neurobiology and neurophysiology to evolution. In addition to the obvious influence exerted by evolutionary processes on neural ontogenesis and on neurobiological mechanisms [57], this novel relationship stems from the understanding that both the construction of the nervous system and its operation are continuously scrutinized for their efficacy in enabling the organism to cope with environmental demands. Hence, the notion that neural development and plasticity represent the biological substrates of adaptation has led to propose that these processes are regulated by fundamental mechanisms that are shared with Darwinian evolution and, notably, the mechanisms of natural selection $[8,13]$.

This concept originated from the discovery that some fundamental ontogenetic phenomena, such as the formation of appropriate numbers of neurons or synapses in the brain, are subjected to environmental constraints, in a way that is reminiscent of the regulation of population size in living organisms. For instance, there is now general agreement that most neuron populations are initially generated in excess and attain their final numbers by a process of cell elimination, in which death or survival depend on the extension of innervation territories, the availability of targetderived trophic substances or the level of neuronal activity [27, 47]. Similar considerations are usually applied to synaptogenesis, where initially exuberant contacts are progressively withdrawn according to a set of restrictive parameters, including levels of activity, spatio-temporal patterns of synaptic activation or activity-dependent uptake of neurotrophic factors [27, 62].

This large body of evidence highlights the role of selective mechanisms in aspects of neural development and plasticity that are strictly related to adaptation. Nevertheless, a purely selective mechanism implies a range of pre-existing potentialities, which is restricted following confrontation with intervening demands. In other words, all the available options should be hardwired ex ante in the ontogenetic program responsible for constructing the organism. Now, is such a mechanism really compatible with adaptation? How can the variety of pre-existing potentialities be expanded at an adequate pace to match the speed of environmental change? Are the discarded options permanently lost or can they be rescued if they become again advantageous in the future?

A selective strategy is primarily designed to control adaptation at the population level. Hence, it is most efficient in regulating species evolution or, as we will discuss later, in defining the number of neurons belonging to a certain category. On the other hand, the main goal of neural adaptation is to allow individual organisms to cope with changing environmental conditions. A closer examination of neural development and plasticity in this perspective actually suggests that the nervous 
system must be endowed with an intrinsic capability to construct neural circuits so 72 to create novel functional properties, beyond the original set of potentialities. As a 73 consequence, both selective and constructive mechanisms participate to determine 74 neural ontogenesis and plasticity. Constructive strategies, however, prevail over 75 selective ones when the individual nervous system has to face contextual environ- 76 mental demands.

\section{Adaptive Mechanisms Can Be Either Predictive or Reactive}

Biological modifications set up to cope with environmental changes occur according to two main modes. On one side, the organism is able to predict the incoming variation and builds up an anticipated response. On the other, the organism is unable to foresee the external change and it can only react to novel conditions once they have been established. Thus, predictive adaptation implies that the organism is ready to face the novel environmental demand at the time when it materializes, whereas reactive adaptation will be only unfolded in a subsequent time.

At a first glance, predictive adaptation may appear more efficient in favouring survival of the organism. Nonetheless, it can be only used in a restricted set of situations. Actually, predictive mechanisms are only suitable to face extrinsic changes that happen at a constant pace through a long period of time (essentially forever). Organisms that spontaneously acquire predictive abilities are favoured over their counterparts and, hence, these abilities become selected by evolutionary mechanisms. Accordingly, predictive adaptation is usually sustained by highly conserved gene networks, whose spatio-temporal patterns of activation correspond to the time course or space distribution of the related environmental conditions. The best example of this kind is the regulation of circadian and circannual functions [12, 19]. These functions are operated by molecular cascades endowed with intrinsic rhythms that match the duration of relevant environmental periods, to which they become entrained by sensory information. As we will discuss here, predictive mechanisms operate in some major ontogenetic processes, which are also governed by highly conserved gene programs. For instance, the gene networks that direct the building of the body (and neural) plan have clearly evolved to cope with consistent environmental constraints, such as gravity, the sources of energy or relevant sensory information (e.g. sunlight) or the mechanics of movement.

Albeit successful, predictive strategies take very long times to become established and diffused. In addition, it is clear that the vast majority of environmental changes happen according to completely unpredictable frequencies and locations. Such situations can be adequately faced only by means of reactive processes, which allow individual organisms or populations to design and set up novel responses. In these cases, evolutionary processes favour the emergence and maintenance of certain abilities, but leave ample degrees of freedom in their actual expression. Most homeostatic mechanisms work in this way. For instance, body temperature is maintained by a series of evolutionary-selected interdependent 
devices, from thyroid hormones to horripilation, whose function is triggered and modulated by feedback loops that tune every response to the concomitant situation.

The vast majority of external conditions that may influence the function of the nervous system belong to the latter category. More, I would say that the main emerging property of the nervous system is to design novel strategies to solve unprecedented problems. Accordingly, neural cells and circuits must be endowed with the ability of reshaping connectivity so to generate new functional capabilities that are not part of the constitutive repertoire of the species. Acquiring new information or learning new skills are examples of this sort of morpho-functional modification that underlies neural adaptation. Hereafter, I will argue that these processes, that are crucial to regulate neural development and plasticity, cannot be solely explained in terms of selective mechanisms, but require constructive properties that allow the creative design of new adaptive strategies.

\section{Neural Development and Evolutionary Mechanisms}

In the perspective of this essay, neural development can be schematically subdivided in three main phases (Fig. 1): (1) neurulation refers to the formation of the neural tube and its segmentation into discrete morphogenic regions; (2) neurogenesis is the process by which neurons (and glia) are generated; (3) synaptogenesis is the process by which neurons become connected to each other into functional circuits. These phases comprise both addition (e.g. generation of new neurons, formation of new synapses) and loss of elements (e.g. physiological cell death, synaptic pruning). Therefore, the growth of the nervous system actually results from the balance of concurrent expansive and regressive phenomena.

Neurulation is triggered by inductive signals issued by the notochord, a mesodermal structure lining the rostro-caudal axis of the embryo, which triggers profound morphogenic rearrangement of the overlying ectoderm leading to the formation of the neural tube $[3,27]$. The latter is a highly polarised structure, which soon becomes subdivided in discrete domains that acquire distinctive morphofunctional specification along the main spatial axes (Fig. 1) [3, 27]. The most important partition occurs along the rostro-caudal axis, where morphologically distinct segments appear, corresponding to the major subdivisions of the adult Central Nervous System (CNS). Within each of such segments, the dorso-ventral axis defines sensory or motor structures, whereas the medio-lateral axis defines the relationship linking neural circuits to axial structures (the trunk) and distal appendages (the limbs).

The regionalization and spatial specification of the neuraxis are determined by the interplay between diffusible or contact signalling cues and the combinatorial expression of specific sets of transcription factors [3]. The whole process is regulated by gene networks, which direct the morphogenesis of the entire body plan. This gene program has been particularly successful during evolution: it has been inherited from invertebrates and it is highly conserved through the phyla of 


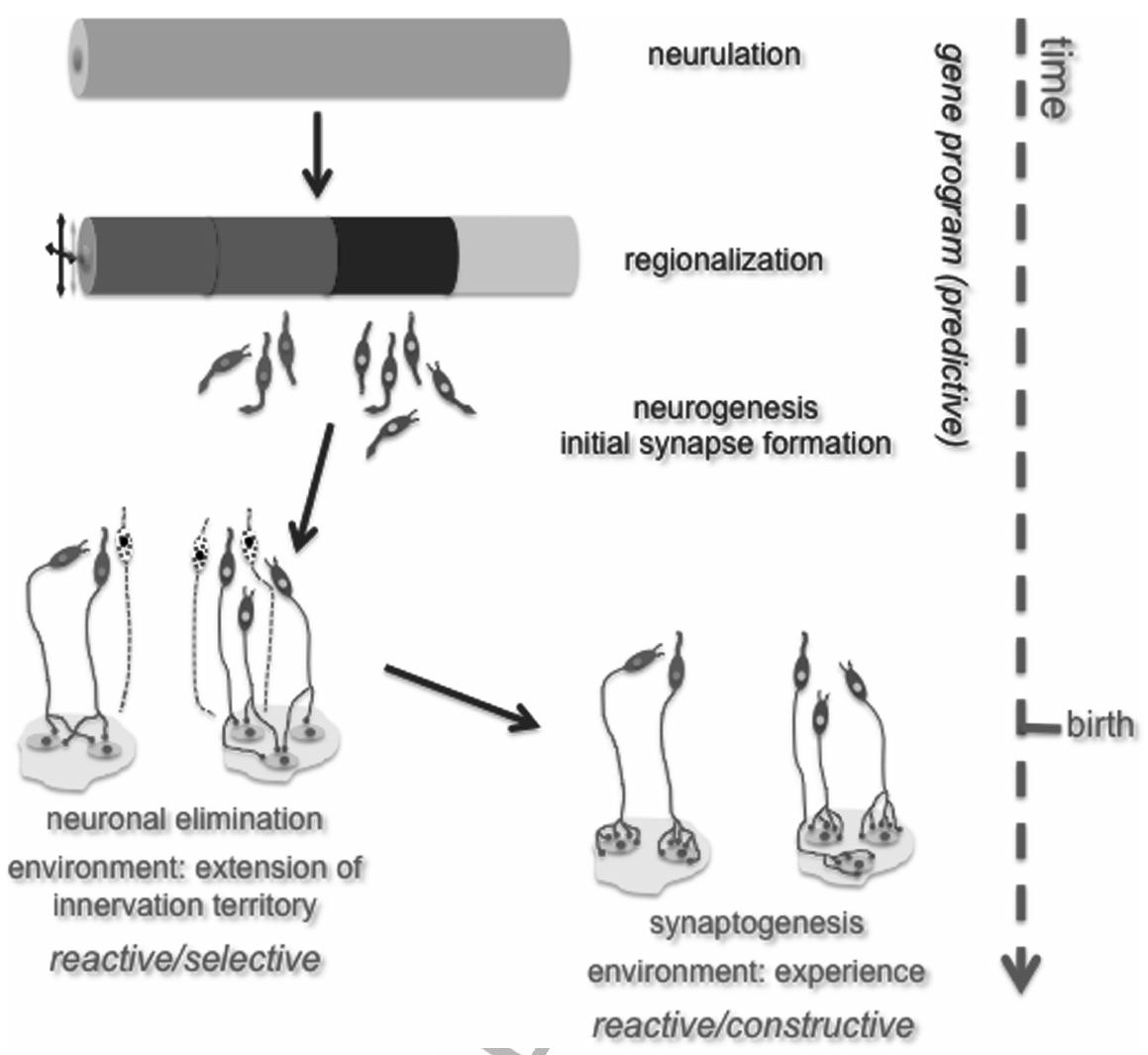

Fig. 1 Regulatory mechanisms of neural ontogenesis. The early phases of nervous system development are determined by the execution of a gene program that directs neurulation, the regionalization of the neural tube, the generation of nerve cells and the initial formation of synapses. While these processes are regulated in a predictive manner, later phases are accomplished according to reactive strategies, required to adapt ontogenetic processes to contextual environmental conditions. Surplus neurons are eliminated before birth by a selective mechanism depending on the extension of available innervation territories. On the other hand, synaptogenesis is carried out after birth, when the organism is interacting with the external world. Hence, synapse formation and reshaping are governed by experience-dependent constructive mechanisms

vertebrates [51]. The program assembles a structural scaffold, in which fundamental morphogenic interactions are precisely regulated in space and time, securing the coordinate development of intrinsic neural networks and their appropriate integra15
15 tion within the nascent organism. On this basic canvas, evolution creates diversity by introducing domain-specific variations in the rate of growth and in the connection patterns. In this way, birds have a relatively large mesencephalon, whereas mammals are characterized by a prominent telencephalon. Thus, neural morphogenesis is accomplished, in a predictive manner, by the intrinsic activity of specific gene networks, whose success is determined a posteriori by natural selection. 
Neurogenesis, which is obviously interrelated with morphogenesis, comprises all the phenomena leading to the generation of neurons and glia from neural stem and progenitor cells (Fig. 1) [27]. These cells proliferate in germinal structures located at different levels along the neuraxis, become specified towards different identities and migrate to specific locations, where they acquire mature phenotypes. Then, the final size of each neuronal population can be refined through physiological cell death. The generation of phenotypic diversity is largely determined by diffusible molecular cues or cell-to-cell interactions that regulate the expression of particular combinations of transcription factors [14, 22, 37, 39]. Once cell fate choices have been taken, however, the differentiation into mature phenotypes is achieved by the unfolding of type-specific gene programs, in an essentially cellautonomous manner. Hence, neuronal differentiation as well as the establishment of the basic framework of connectivity are also governed by predictive mechanisms that determine a priori the capability of a given neuron to migrate into a certain position, orientate the navigation of its axon or recognize appropriate targets.

The situation is different when the regulation of neuron numbers is considered (Fig. 1). The number of neurons generated for each category is determined by the interplay between intrinsic properties of neural progenitors and local regulatory interactions that modulate the rhythm of proliferation, the relative proportion of cells that initiate differentiation or continue to divide, and the duration of neurogenic periods [7,33]. All these mechanisms operate to regulate neuron numbers by adjusting their production and, hence, work according to a predictive strategy. Nevertheless, since the pioneering work of Rita Levi-Montalcini and Giuseppe Levi [30], it is well known that most neuron populations are actually generated in excess and the final amount of nerve cells that populate the mature nervous system is achieved through the elimination of supernumerary elements [42]. Cell death or survival depend on a set of parameters, including both intrinsic features of the neurons (e.g. their level of activity) and environmental constraints (e.g. the extension of the target field or the availability of neurotrophic substances). This process is suitable to match the size of each neuronal population to the amount of potential synaptic partners or to the extension of innervation territories in the periphery. It operates according to a selective mechanism that is most reminiscent of natural selection: the juvenile neurons compete for limited quantities of available resources and their fate depends on their intrinsic ability to overcome their rivals [8, 47]. In this case, however, the mechanism works following a reactive strategy, required to adjust neural development to individual fluctuations in the dimension of different parts of the body. Accordingly, the size of most neuron populations can be significantly modified by experimental manipulations that increase or reduce the extension of the available innervation territory $[27,42,44]$. Therefore, the final number of neurons belonging to each population derives from a dual mechanism, which combines a predictive component, that determines the initial production of surplus neurons, and a reactive component, that eliminates supernumerary elements in response to contextual environmental conditions.

At a first glance, similar mechanisms may apply during synaptogenesis (Fig. 1). A well-established notion in developmental neurobiology is that synapses are 
initially formed in excess and then partially withdrawn to shape the mature connec- 20 tivity [27, 47]. Since effective function of neural circuits depends both on the 20 number and on the specificity of synapses, the pruning process would be required 21 both to reduce the exuberant, supernumerary contacts and to remove aberrant, 21 wrong connections.

The initial formation of synapses is guided by recognition cues exposed on the 21 : neuronal membrane, whose nature is determined by the intrinsic neurochemical 21 profile of the partner neurons $[5,61,66]$. Synaptic pruning is driven by activity- 21 dependent mechanisms that are directly influenced by the functional efficacy of the 21 developing circuitry $[8,47]$. Thus, synaptogenesis also appears to depend on a dual 21 mechanism. Synapse formation is guided by molecular interactions determined by 21 the unfolding of neuronal-intrinsic gene programs that work in a predictive manner. 21 On the other hand, synaptic pruning is driven by an essentially reactive mechanism 22 that selects good connections on the basis of their functional meaningfulness. Again, 22 the latter phenomenon appears to follow some fundamental principles of natural 22 selection.

The analogy is partial at best. It is well established that a number of synapses are 22 withdrawn to shape appropriate spatial connection patterns on specific target 22 domains. Nonetheless, it is definitely clear that, when the number of contacts and/ 22 or their functional weight is considered, the final balance of the synaptogenic 2 process is a positive one: newly-formed synapses greatly outnumber the lost ones 22 $[46,49,62]$. This has been clearly demonstrated in a variety of experimental 22 models, including the autonomic nervous system [31, 48], the visual system [60], 23 or the cerebellar climbing fibres [23], just to cite a few ones. Even in the case of the 23 neuromuscular junction where mono-innervation of muscle fibres appears to be 23 solely achieved through the elimination of supernumerary axons, the winner 23 endplate undergoes a remarkable outgrowth to cover the entire postsynaptic surface with additional junctional complexes and releasing sites [46, 55]. Therefore, the reactive component of synaptogenesis is not a selective process, but rather operates in a constructive manner.

This conclusion has profound implications in terms of structure-to-function 23 relationship during neural development. Indeed, while the initial formation of 23 synaptic contacts is essentially aimed at establishing a basic framework of neural networks capable of initiating the interaction with the external world, the refinement phase is aimed at modifying the structure of such networks to improve their 24 operational abilities. Thus, a fundamental circuit scaffold, assembled by executing 24 intrinsic gene programs, is confronted with experience and modified to achieve 24 adaptive function. The latter process involves the elimination of some unspecific 24 contacts, but it is primarily characterized by the strengthening of meaningful connections with the addition of numerous new synapses.

This process of structural remodelling, which involves the simultaneous 24 outgrowth of both presynaptic axons and postsynaptic dendrites [44, 48], leads to 24 the emergence of novel functional properties, whose nature is influenced by the 25 specific features of the contextual environmental conditions. In other words, the 25 final structure of neural circuits is congruent with the actual experience: a particular 25 
interaction with the external world will always lead to an appropriate pattern of connectivity [53]. The essentially constructive nature of this process can be best appreciated in extreme experimental conditions. For instance, severe manipulations such as monocular deprivation or experimental squid during the critical periods of visual system development induce extensive changes in the connectivity of the

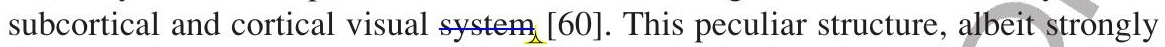
divergent from that of the normal population, is clearly adaptive when the visual experience of the relevant individuals is considered. Indeed, there is no reason to leave half of the cortical territory to an eye that is not conveying any significant sensory information. Similarly, there is no use to form binocular connections if the two eyes are seeing different scenes. Yet, it is difficult to believe that such unusual projection patterns result from the selection of pre-existing connections, rather than being actively constructed by adapting the morpho-functional properties of the circuit to real life experience. Similar considerations apply to other systems, such as the peculiar tonotopic representation that can be induced in the auditory cortex by exposure to auditory stimuli of specific frequencies [10].

On the whole, the initial phases of nervous system development, which include neural morphogenesis, neuronal production and the establishment of basic connection patterns, are directed by the activity of species-specific gene networks that operate according to an essentially predictive strategy. These processes lead to assemble the fundamental framework of the nervous system, which then undergoes individual-specific morpho-functional adaptation according to reactive strategies. Neuron numbers are refined through a primarily selective process, whereas synaptic patterns are reshaped according to constructive mechanisms. The latter mechanisms have been likely evolved to exploit influences derived from contextual experience to favour the development of adaptive function.

\section{Experience-Dependent Mechanisms, Neural Development and the Emergence of Function}

A major feature of the last phases of neural development is the appearance of reactive processes that essentially shift adaptation from species to individuals. Such processes, however, are accomplished during distinct ontogenetic phases, characterized by strongly different conditions [27, 46]. Neurogenesis and physiological cell death primarily occur before birth and are influenced by somatic changes taking place within the same developing organism. On the other hand, the bulk of synaptogenesis is carried out after birth, while the newborn organism is actively interacting with the external world. The latter condition exerts a most dramatic influence on the course and on the outcome of this process.

Higher vertebrates, notably mammals, are born with immature neural circuits, and this feature is most prominent in primates and humans [45, 57]. This implies that crucial phases of neural development occur while the organism is exposed to 


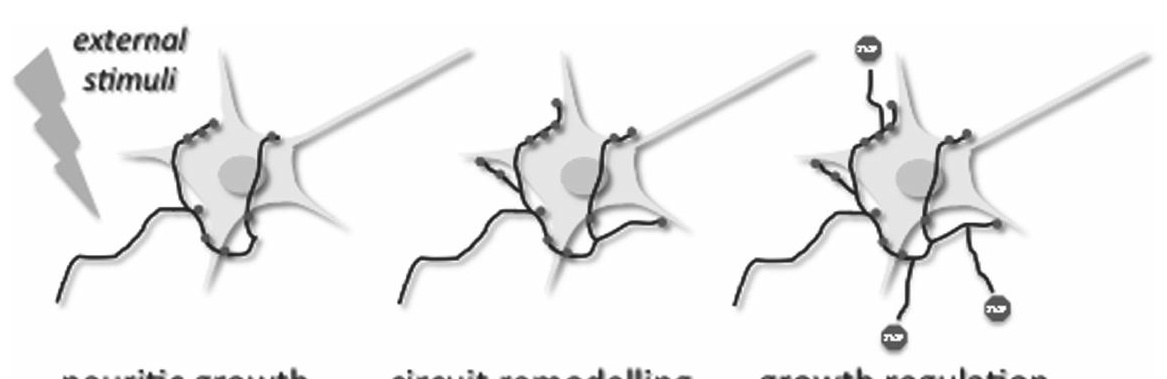

neuritic growth circuit remodelling growth regulation

Fig. 2 External stimuli direct developmental synaptogenesis and adult circuit plasticity. External stimuli drive plastic modification of neural circuits by inducing neuritic remodelling and directing the formation of functionally meaningful contacts. The process is regulated by inhibitory cues present in the CNS microenvironment (represented by the STOP signals), required to prevent aberrant growth and dysfunction

the external environment rather than sheltered in an egg or in the uterus. The 29 newborn CNS, and particularly those structures that are more immature at birth 29 such as the neocortex, is subjected to a wide range of powerful stimuli, which 29 induce specific patterns of neuronal activation, stimulate neuritic extension and 29 influence the number and the distribution of newly-formed synapses [63]. This 29 ability of experience to stimulate neural growth is the crucial event that shifts the 29 nature of synaptogenesis from a selective process aimed at achieving synaptic 29 specificity to a constructive one capable of building new functionally meaningful 30 connections (Fig. 2).

Sensory deprivation experiments, such as dark rearing or exposure to un- 30 modulated acoustic stimulation $[11,60,65]$, show how administration of meaningful 30 stimuli immediately activates neuronal growth mechanisms, associated with rapid 30 . acquisition of new functional properties. All these examples of experience-dependent 30 structural remodelling are characterized by a clear prevalence of expansive phenom- 30 ena, with the formation and strengthening of new synapses, over regressive events and 30 loss of contacts. Hence, experience drives neuronal growth to create adaptive function. 30 The evolutionary advantage of this strategy is obvious: each individual organism 30 capable of exploiting contextual experience to generate appropriate novel responses will be able to successfully cope with a wide range of unprecedented situations.

Once function is acquired, synaptogenic processes are greatly reduced if not 31: completely arrested [24]. This decline of neuronal growth properties, that marks the 31 end of developmental critical periods for the acquisition of experience-dependent 31 capabilities, has been attributed to a set of concurrent mechanisms. The remodelling of neural circuits often leads to a substantial segregation of afferent axons, which 31 impinge upon private target domains, being individual dendrites, single neurons or 31 discrete anatomical modules. This process of input segregation would progressively 31 reduce the need and the opportunity for activity-dependent competitive interactions that sustain synaptogenesis [62]. Hence, growth would be arrested when a stable 32 connection pattern is achieved and all partners had their share. 
In spite of the attractive simplicity of this mechanism, the end of synaptogenic processes is actually coincident with profound modifications that occur in the neurons themselves and in the surrounding microenvironment [53]. Within the nerve cells, growth-associated gene programs are actively suppressed to favour information processing and signalling function. Coincidentally, the maturation of glia, namely myelination, and the deposition of the extracellular matrix are accompanied by the appearance of a variety of growth-inhibitory molecules that stabilize contacts and hamper further elongation of neuronal processes (Fig. 2). These phenomena are precisely aimed at restricting growth properties of neural circuits. As we will see in the next section, synaptogenic properties typical of juvenile organisms can be restored in the mature CNS by specific manipulations that boost intrinsic neuronal growth properties or remove environmental inhibition.

The presence of such strict growth control mechanisms, which have been progressively implemented during the evolution of vertebrates [17, 56], represents an additional argument favouring the constructive nature of developmental synaptogenesis. Indeed, a purely selective mechanism is self-limiting and does not require additional regulatory devices to be terminated. On the contrary, a constructive mechanism must be actively arrested, either by removing the sustaining stimuli or by dampening growth processes. Experience cannot be prevented or abolished: the whole ontogenetic process is precisely aimed at making the nervous system able to cope with external constraints. Therefore, when the development of neural circuits adopted the constructive strategy driven by experience-dependent stimulation, a set of growth-inhibitory mechanisms evolved to stabilize meaningful connections and to restrain neuronal growth once function is achieved. Not surprisingly, the induction of such regulatory molecules is also triggered by experience $[26,59]$.

\section{Constructive Mechanisms and Plasticity in the Adult}

In spite of the clear decline of intrinsic neuronal growth potentialities, after the end of canonical ontogenesis the nervous system retains a certain degree of ability to modify his structure and function in response to external stimuli or changes in the environment. Adaptation in the mature nervous system, which is generally known as plasticity, shares some fundamental features and mechanisms with developmental processes. The notion of plasticity in the adult CNS was established several decades ago with the discovery of reactive synaptogenesis and synaptic turnover $[9,50]$. Accordingly, for a long time the adaptive abilities of neural circuits were thought to be exclusively sustained by changes of connectivity. Recently, however, the demonstration that neurogenesis persists at least in some regions of the adult mammalian brain has revealed that functional adaptation can be also carried out by integrating new neurons in pre-existing circuits.

Compared to neural development, synaptogenic phenomena occurring in the adult nervous system are considerably restricted in space and time. They involve 
both formation and withdrawal of synaptic contacts and, although they usually lead 36 to moderate changes of synaptic numbers, they obey to reactive mechanisms and 36 have a clear constructive character. One important difference with juvenile 36 synaptogenesis is the requirement of active participation [29, 64]. Synaptic 36 remodelling in immature organisms is usually triggered by the mere exposure to 36 external stimuli. In contrast, in adulthood plastic changes also require motivation 36 and active participation of the involved organism. Hence, in mature individuals adaptation is no more an automatic response to environmental conditions, but 37 requires an individual volition that determines the nature of the response and 37 influences its outcome.

Plasticity in the adult is strongly hampered by the presence of the above- 37 mentioned inhibitory mechanisms that terminate developmental synaptogenesis. 37 These mechanisms are partially counteracted by the growth promoting effect 37 exerted by external stimuli $[18,20,54]$. Accordingly, structural plasticity and 37 functional adaptation in the adult can be conspicuously enhanced by experimental 37 procedures that activate neuronal growth genes or neutralize inhibitory molecules 37 of the CNS microenvironment [53]. Nevertheless, whatever effective the simple 37 manipulation of the molecular devices that control neuritic growth is not sufficient 38 to induce adaptation. Endurable structural changes associated with significant 38 functional modifications can only be established if these procedures are combined 38 with specific environmental stimuli [43]. Hence, growth regulatory mechanisms exert a purely permissive role by setting the degree of plasticity of neural circuits, 38 whereas environmental stimulation has a primarily instructive function in determining the shape of the connectivity that will be formed [53].

These features are consistent with a reactive mechanism that induces structural 38 remodelling of neural circuits to generate adaptive responses. As for developmental 38 synaptogenesis, the presence of multiple inhibitory mechanisms is required to 38 maintain constructive modifications within the limits of adaptive function. Indeed, 39 there are several examples showing that altered regulatory mechanisms and/or 39 unusual experience may induce unspecific growth associated with frank pathological 39 phenomena, such as seizures or dystonia $[1,6,40]$. A selective mechanism may fail 39 to generate an adaptive response if the required option is not available, but it should 3 be intrinsically unable to produce abnormal structures and aberrant function. Thus, 3 plasticity in the adult also follows a constructive strategy and, for this reason, it 3 must be subjected to inhibitory control.

Adult neurogenesis shares its major functional significance with adult plasticity. In some CNS structures adaptation is not exclusively sustained by changes of 3 connectivity, but also involves the integration of newly generated neurons into 40 pre-existing circuits. As discussed above, developmental neurogenesis comprises a 40 predictive mechanism that generates excessive amounts of neurons, whose final 40 number is defined by a reactive mechanism that operates through selection. The 40 scenario of adult neurogenesis is very different. In both regions of mammalian brain where new neurons are generated throughout life, the hippocampal dentate gyrus 40 and the olfactory system, the rate of neuronal generation is clearly influenced by 40 external stimuli and/or activity-dependent mechanisms $[15,35]$. Thus, while the 40 
adult system retains the capacity for generating neurons, the course and outcome of the process are no more determined by an intrinsically-coded predictive mechanism, but regulated by extrinsic cues according to a reactive strategy.

Many of the newly generated neurons survive only for a short time, suggesting that survival may depend on selective mechanisms, as for developmental neurogenesis. However, the number and the specific features of the neurons that eventually become stably integrated in adult circuits depend on the activity of the involved network and on specific functional demands [2, 28, 32, 41]. In other words, integration of the newborn neuron is directly related to the function that is being established and not to the intrinsic receptive capacity of the system. Therefore, similar to synaptic remodelling, adult neurogenesis appears to work as a reactive device obeying to a primarily constructive strategy.

This conclusion is further supported by the observation that neurogenesis, or at least neurogenic attempts, may be induced in other regions of the CNS by strong stimulation or pathological conditions $[4,34,52,58]$. In these instances, nonneurogenic structures react to extreme environmental constraints by redirecting the specification of local progenitors towards neuronal lineages. These phenomena of intraparenchymal neurogenesis are often abortive, because non-neurogenic regions fail to provide adequate conditions to support the differentiation and integration of new neurons. Hence, latent neurogenic potentialities may be diffused in many CNS regions, but actively repressed by local constraints. In any case, adult neurogenesis appears to be driven by environmental stimuli influencing the mature tissue, rather than local regulatory cues acting in a primary germinal structure.

Another feature that adult neurogenesis shares with adult plasticity is the presence of strict inhibitory control. Intrinsic inhibitory control prevents adult neurons from de-differentiating or re-entering the cell cycle [25]. In addition, environmental cues regulate the proliferation of progenitors as well as the migration, differentiation and integration of newborn neurons [38]. Thus, successful incorporation of new neurons in adult networks is restricted to precise phenotypes in defined circuits. Furthermore, transplantation experiments show that the endogenous ability of the adult CNS to accommodate donor neurons in functional circuits is limited to a few types and locations $[21,36]$. These inhibitory constraints also appear to be primarily aimed at preventing aberrant phenomena that may lead to maladaptive function or behaviour. However, these considerations indicate that adult neurogenesis also has the main characters of a reactive/constructive process, in which experience-dependent growth is exploited to modify neural structures so to achieve adaption.

\section{Conclusions}

The initial phases of neural development are primarily regulated by predictive mechanisms that have been established by evolution. These processes, which are highly conserved throughout vertebrate phylogenesis, are designed to develop a nervous system that is suitable to control the main bodily functions of the organism 
and is capable of interacting with the external world. The sensitivity of neural 44 circuits to external stimuli, however, profoundly influenced the strategy of neural 45 development. When coping with rather constant phenomena, such as the physio- 45 logical expansion or retraction of different body parts, suitable adaptation can be 45 obtained by merely selective mechanisms, which share some features with natural 45 selection. Hence, neurogenesis starts with the production of surplus neurons and 45 their final number is adjusted to match actual requirements, which may fluctuate 45 among individuals, but always remain within predictable ranges. A similar mecha- 45 nism may also apply to synaptogenesis if the nervous system was designed to be completely hardwired by intrinsic genetically-determined mechanisms.

Quite surprisingly, however, the exposure of the immature nervous system to the external environment dramatically changed the ontogenetic strategy. Now, the ability of coping with a great variety of unpredictable environmental constraints could not be adequately fulfilled by a selective process. Rather, the expanding variety of situations favoured the emergence of an alternative mechanism, able to create unprecedented structure and function to face unprecedented situations. Thus, evolutionary pressure pushed developmental synaptogenesis, adult plasticity and even adult neurogenesis to become reactive processes obeying to the rules of constructive mechanisms. This constructive revolution of neural ontogenesis induced the appearance of specific regulatory mechanisms, which evolved to restrain the unchained growth driven by external stimuli within the limits of 4 adaptive function. These inhibitory cues first appeared in fish and amphibians 47 [56], but their importance consistently increased during later vertebrate evolution, 47 in parallel with the increasing complexity of CNS structure and function. Now, they 47 clearly fulfil the fundamental task of controlling potentially dangerous growth 47 properties that enable the nervous system of powerful plastic and adaptive capabilities. However, they also bring with themselves some relevant side effects, 47 such as the loss of neural regeneration capabilities $[16,17]$. In any case, constructive mechanisms, such as those directing adult plasticity and neurogenesis, repre- 47 sent a most successful phylogenetic invention that greatly increased the individual 47 ability to cope with increasingly wide ranges of environmental conditions.

Acknowledgements The scientific work of Ferdinando Rossi is supported by grants from Ministero dell'Universită e della Ricerca Scientifica e Tecnologica (MIUR-PRIN 2007 prog. nr. 2007F7AJYJ), Compagnia di San Paolo (Neurotransplant Project 2008; GABAGEN Neuroscience project 2009), Regione Piemonte (Project A14/05; Ricerca Sanitaria Finalizzata, 2008, 2009), Ataxia UK; Fondazione Cavaliere del Lavoro Mario Magnetto of Turin.

\section{References}

1. Aigner L, Arber S, Kapfhammer JP, Laux T, Schneider C, Botteri F, Brenner H-R, Caroni P (1995) Over expression of the neural growth associated protein GAP-43 induces nerve sprouting in the adult nervous system of transgenic mice. Cell 83:269-278

2. Aimone JB, Deng W, Gage FH (2010) Adult neurogenesis: integrating theories and separating functions. Trends Cogn Sci 14:325-337 
3. Bronner-Fraser M, Hatten ME (2003) Neural induction and pattern formation. In: Squire LR, Bloom FE, McConnell SK, Roberts JL, Spitzer NC, Zigmond MJ (eds) Fundamental neuroscience. Academic, New York/London

4. Brundin P, Winkler J, Masliah E (2008) Adult neurogenesis in neurodegenerative diseases. In: Gage FH, Kempermann G, Song H (eds) Adult neurogenesis. Cold Spring Harbor Laboratory Press, Cold Spring Harbor, pp 503-533

5. Burden SJ, Berg D, O'Leary DDM (2003) Target selection, topographic maps and synapse formation. In: Squire LR, Bloom FE, McConnell SK, Roberts JL, Spitzer NC, Zigmond MJ (eds) Fundamental neuroscience. Academic, New York/London

6. Byl N, Merzenich MM, Jenkins WM (1996) A primate genesis model of focal dystonia and repetitive strain injury. I. Learning-induced dedifferentiation of the representation of the hand in the primary somatosensory cortex in adult monkeys. Neurology 47:508-520

7. Caviness VS Jr, Nowakowski RS, Bhide PG (2009) Neocortical neurogenesis: morphogenetic gradients and beyond. Trends Neurosci 32:443-450

8. Changeux JP (1983) L'homme neuronal. Fayard, Paris

9. Cotman C, Sampedro N, Harris EW (1981) Synapse replacement in the nervous system of adult vertebrates. Physiol Rev 61:684-784

10. De Villers-Sidani E, Chang EF, Bao S, Merzenich MM (2007) Critical period window for spectral tuning defined in the primary auditory cortex (A1) in the rat. J Neurosci 27:180-189

11. De Villers-Sidani E, Simpson KL, Lu Y-F, Lin RCS, Merzenich MM (2008) Manipulating critical period closure across different sectors of the primary auditory cortex. Nat Neurosci 11:957-965

12. Dunlap JC, Loros LJ, De Coursey P (2004) Chronobiology: biological timekeeping. Sinauer, Sunderland

13. Edelman G (1987) Neural Darwinism. The theory of neuronal group selection. Basic Books, New York

14. Edlund T, Jessell TM (1999) Progression from extrinsic to intrinsic signaling in cell fate specification: a view from the nervous system. Cell 96:211-224

15. Fabel K, Kempermann G (2008) Physical activity and the regulation of neurogenesis in the adult and aging brain. Neuromolecular Med 10:59-66

16. Fawcett JW, Rosser AE, Dunnett SB (2002) Brain damage, brain repair. Oxford University Press, Oxford

17. Ferretti P, Zhang F, O'Neill P (2003) Changes in spinal cord regenerative ability through phylogenesis and development: lessons to be learnt. Dev Dyn 226:245-256

18. Foscarin S, Ponchione D, Pajaj E, Leto K, Gawlak M, Wilczynski GM, Rossi F, Carulli D (2011) Experience-dependent plasticity and modulation of growth regulatory molecules at central synapses. PLoS One 6:e16666

19. Foster RG, Kreitzman L (2004) Rhythms of life: the biological clocks that control the daily lives of every living thing. Profile Books, London

20. Gomez-Pinilla F, Ying, Z, Agoncillo T, Frostig R (2011) The influence of naturalistic experience on plasticity markers in somatosensory cortex and hippocampus: effects of whisker use. Brain Res (in press)

21. Grimaldi P, Carletti B, Rossi F (2005) Neuronal replacement and integration in the rewiring of cerebellar circuits. Brain Res Rev 49:330-342

22. Harris WA, Hartenstein V (2003) Cellular determination. In: Squire LR, Bloom FE, McConnell SK, Roberts JL, Spitzer NC, Zigmond MJ (eds) Fundamental neuroscience. Academic, New York/London

23. Hashimoto K, Hichikawa R, Kitamura K, Watanade M, Kano M (2009) Translocation of a "winner" climbing fiber to the Purkinje cell dendrite and subsequent elimination of "losers" from the soma in developing cerebellum. Neuron 63:103-118

24. Hensch T (2004) Critical period regulation. Annu Rev Neurosci 27:549-579

25 . Herrup K, Yang Y (2007) Cell cycle regulation in the postmitotic neuron: oxymoron or new biology? Nat Rev Neurosci 8:368-378 
26. Hockfield S, Kalb RG (1993) Activity-dependent structural changes during neuronal development. Curr Opin Neurobiol 3:87-92

27. Jacobson M (1991) Developmental neurobiology. Plenum, New York/London

28. Kempermann G, Fabel K, Ehninger D, Babu H, Leal-Galicia P, Garther A, Sa W (2010) Why and how physical activity promotes experience-induced brain plasticity. Front Neurosci 4:189

29. Keroughlian AS, Knudsen EI (2007) Adaptive auditory plasticity in developing and adult animals. Prog Neurobiol 82:109-121

30. Levi-Montalcini R, Levi G (1944) Correlazioni nello sviluppo tra varie parti del sistema nervoso. Pont Acad Sci 8:527-568

31. Lichtman JW (1977) The reorganization of synaptic connexions in the rat submandibular gnalgion during post-natal development. J Physiol 273:155-177

32. Lledo PM, Saghatelyan A (2005) Integrating new neurons into the adult olfactory bulb: joining the network, life-death decisions, and the effects of sensory experience. Trends Neurosci 28:248-254

33. Lukaszewicz A, Savatier P, Cortay V, Giroud P, Huissoud C, Berland M, Kennedy H, Dehay C (2005) G1 phase regulation, area-specific cell cycle control, and cytoarchitectonics in the primate cortex. Neuron 47:353-364

34. Luzzati F, De Marchis S, Fasolo A, Peretto P (2007) Adult neurogenesis and local neuronal progenitors in the striatum. Neurodegener Dis 4:322-327

35. Ma DK, Kim WR, Ming GL, Song H (2009) Activity-dependent extrinsic regulation of adult olfactory bulb and hippocampal neurogenesis. Ann NY Acad Sci 1170:664-673

36. MacLaren RE, Pearson RA, MacNeil A, Douglas RH, Salt TH, Akimoto M, Swaroop A, Sowden JC, Ali RR (2006) Retinal repair by transplantation of photoreceptor precursors. Nature 444:203-207

37. McConnell SK (1995) Strategies for the generation of neuronal diversity in the central nervous system. J Neurosci 15:6987-6998

38. Ming GL, Song H (2005) Adult neurogenesis in the mammalian central nervous system. Annu Rev Neurosci 28:223-250

39. Muotri A, Gage FH (2006) Generation of neuronal variability and complexity. Nature 441:1087-1093

40. Nudo RJ (2003) Retuning the misfiring brain. Proc Natl Acad Sci USA 100:7425-7427

41. Oboti L, Savalli G, Giachino C, De Marchis S, Panzica GC, Fasolo A, Peretto P (2009) Integration and sensory experience-dependent survival of newly-generated neurons in the accessory olfactory bulb of female mice. Eur J Neurosci 29:679-692

42. Oppenheim RW, Johnson JE (2003) Programmed cell death and neurotrophic factors. In: Squire LR, Bloom FE, McConnell SK, Roberts JL, Spitzer NC, Zigmond MJ (eds) Fundamental neuroscience. Academic, New York//London

43. Pizzorusso T, Medini P, Berardi N, Chierzi S, Fawcett JW, Maffei L (2002) Reactivation of ocular dominance plasticity in the adult visual cortex. Science 298:1187-1189

44. Purves D (1988) Body and brain: a trophic theory of neural connections. Harvard University Press, Cambridge, MA/London

45. Purves D (1994) Neural activity and the growth of the brain. Cambridge University Press, Cambridge

46. Purves D, Lichtman JW (1980) Synapse elimination in the developing nervous system. Science 210:153-157

47. Purves D, Lichtman JW (1985) Principles of neural development. Sinauer, Sunderland

48. Purves D, Snider VD, Voyvodic JT (1988) Trophic regulation of nerve cell morphology and innervation in the autonomic nervous system. Nature 336:123-128

49. Purves D, White LE, Riddle D (1996) Is neural development Darwinian? Trends Neurosci 19:460-464

50. Raisman G, Field PM (1973) A quantitative investigation of the development of collateral reinnervation after partial deafferentation of the septal nuclei. Brain Res 50:241-264 
596 51. Reichert E (2009) Evolutionary conservation of mechanisms for neural regionalization,

597 proliferation and interconnection in brain development. Biol Lett 5:112-116

598 52. Robel S, Berninger B, Götz M (2011) The stem cell potential of glia: lessons from reactive 599 gliosis. Nat Rev Neurosci 12:88-104

600 53. Rossi F, Gianola S, Corvetti L (2007) Regulation of intrinsic neuronal properties for axon 601 growth and regeneration. Prog Neurobiol 81:1-28

602 54. Sale A, Berardi N, Maffei L (2009) Enrich the environment to empower the brain. Trends $603 \quad$ Neurosci 32:233-239

604 55. Sanes JR, Lichtman JW (1999) Development of the vertebrate neuromuscular junction. Annu 605 Rev Neurosci 22:389-442

606 56. Schweigreiter R (2008) The natural history of the myelin-derived nerve growth inhibitor

608 57. Striedter GF (2004) Principles of brain evolution. Sinauer, Sunderland

609 58. Suh H, Weng D, Gage FH (2009) Signaling in adult neurogenesis. Annu Rev Cell Dev Biol $610 \quad 25: 153-175$

611 59. Sur M, Frost DO, Hockfield S (1988) Expression of a surface-associated antigen on Y-Cells in 612 the cat lateral geniculate nucleus is regulated by visual experience. J Neurosci 8:874-882

613 60. Wiesel TN (1982) Postnatal development of the visual cortex and the influence of environ614 ment. Nature 299:583-591

615 61. Williams ME, de Wit J, Ghosh A (2010) Molecular mechanisms of synaptic specificity in 616 developing neural circuits. Neuron 68:9-18

617 62. Wong ROL, Lichtman JW (2003) Synapse elimination. In: Squire LR, Bloom FE, McConnell 618 SK, Roberts JL, Spitzer NC, Zigmond MJ (eds) Fundamental neuroscience. Academic, New York/London

63. Zheng D, Purves D (1995) Effects of increased neural activity on brain growth. Proc Natl Acad Sci USA 92:1802-1806

64. Zhou X, Merzenich MM (2009) Developmentally degraded cortical temporal processing restored by training. Nat Neurosci 12:26-28

65. Zhou X, Ngarajan N, Mossop BJ, Merzenich MM (2008) Influences of un-modulated acoustic inputs on functional maturation and critical-period plasticity of the primary auditory cortex. Neuroscience 154:390-396

66. Zipursky SL, Sanes JR (2010) Chemoaffinity revisited: dscams, protocadherins, and neural circuit assembly. Cell 143:343-353 


\section{Author Queries}

Chapter No.: 10

\begin{tabular}{|c|c|c|}
\hline Query Refs. & Details Required & Author's response \\
\hline AU1 & $\begin{array}{l}\text { Please update the reference Gomez- } \\
\text { Pinilla et al. (2011). }\end{array}$ & $\begin{array}{l}\text { Brain Res } \\
1388: 39-47\end{array}$ \\
\hline
\end{tabular}

